\title{
ORIGINAL ARTICLE \\ Clinical features and inpatient rehabilitation outcomes of infection-related myelopathy
}

\author{
ML Brubaker ${ }^{1}$, MT Luetmer $^{2}$ and RK Reeves ${ }^{1}$
}

Study design: This was a retrospective cohort study.

Objectives: The objectives of this study were to determine clinical features of infection-related myelopathy (IRM) and functional outcomes compared with other nontraumatic and traumatic myelopathies.

Setting: US academic inpatient rehabilitation unit.

Methods: This was a 16-year retrospective review of patients with myelopathy discharged from inpatient rehabilitation between 1 January 1995 and 31 December 2010. Patients comprised three injury groups: IRM, nontraumatic myelopathy (NTM) and traumatic spinal cord injury (TSCl). Information collected includes demographic characteristics, functional data, length of stay, injury completeness and discharge destination. Primary outcome measures were change in Functional Independence Measure (FIM) and daily FIM change. For IRM, data were collected regarding injury characteristics, risk factors, presenting symptoms, neurologic impairment level and treatment.

Results: Of the 1601 patients, 40 (2.5\%) had IRM, 1105 (69.0\%) had NTM and 456 (28.5\%) had TSCI. IRM mean (s.d.) age was 58.6 (15.7) years (male gender, 72.5\%). The majority in each group had incomplete injuries. IRM had longer lengths of stay $(P<0.001)$, lower admission $(P=0.001)$ and discharge $(P=0.005)$ FIM scores and lower FIM daily change $(P=0.002)$ than NTM. Degree of functional improvement was similar in all groups, and most patients in each group were discharged home. Infectious pathogens were bacterial $(80.0 \%, n=32)$, viral $(7.5 \%, n=3)$, tuberculous $(7.5 \%, n=3)$, parasitic $(2.5 \%, n=1)$ and multiple types $(2.5 \%, n=1)$. The most common bacterial cause $(65.6 \%)$ was Staphylococcus aureus.

Conclusions: Infectious etiologies comprise a small proportion of NTM but represent a unique entity with distinct recovery patterns and outcomes. These patients can achieve similar functional improvements as other NTM patients during inpatient rehabilitation, although over a longer period of time.

Spinal Cord (2017) 55, 264-268; doi:10.1038/sc.2016.115; published online 2 August 2016

\section{INTRODUCTION}

Infectious myelopathies represent a small, but important, subset of the nontraumatic myelopathies (NTMs) requiring inpatient rehabilitation. Although the exact incidence is unknown, studies have found that infection-related myelopathy (IRM) represents between 3\% of patients in developed countries, such as the United States, and $49 \%$ of patients in developing countries with NTM who are admitted to inpatient rehabilitation units worldwide. ${ }^{1-5}$ This variation is largely because of geographic and environmental factors, different health systems and access to health care. ${ }^{4,6,7}$ IRM involving spinal epidural abscesses or tuberculosis is thought to cause injury through several mechanisms, including direct compression of the spinal cord, vascular occlusion causing ischemic injury and vertebral collapse with associated myelitis, whereas viral infections involve a more diffuse neural injury process. $^{8-10}$

IRM appears to affect more men than women and typically occurs during the fourth through seventh decades of life.6,11-16 Studies performed in the United States, Spain, Australia, Singapore and Israel have shown that the most common cause of IRM is epidural abscess, ${ }^{6,16}$ with Staphylococcus aureus being the most commonly

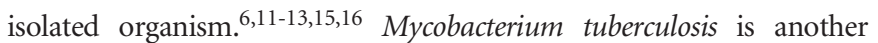
common pathogen found to be a more prevalent cause of infectious myelopathy in Africa, Singapore and India, accounting for 30\%, 46\% and $25 \%$, respectively, of reported IRM. $5,11,17-20$

Although the literature regarding rehabilitation outcomes of NTM is becoming more robust, it continues to be suboptimal for IRM. Of the published rehabilitation literature regarding this population, the methods and outcomes vary widely. The incidence of infectious etiologic factors varies across health-care systems throughout the world, making it difficult to extrapolate one center's results to apply to another. Infections represent a small proportion of the NTMs compared with other more common causes, such as vertebral spondylosis, spinal tumors and vascular ischemia, making this population challenging to study. Early surgical intervention can also affect patient's functional motor outcomes; ${ }^{7,21-23}$ however, it is unknown what percentages of these patients require rehabilitation after acute hospitalization. This lack of consistent data makes it difficult to properly counsel patients, families and medical providers on the expected clinical course and outcomes of infectious myelopathies.

${ }^{1}$ Department of Physical Medicine and Rehabilitation, Mayo Clinic, Rochester, MN, USA and ${ }^{2}$ Mayo Medical School, Mayo Clinic College of Medicine, Rochester, MN, USA Correspondence: Dr ML Brubaker, Department of Physical Medicine and Rehabilitation, Mayo Clinic, Rochester, MN 55905, USA.

E-mail: mbrubaker@craighospital.org

Portions of this manuscript were presented at the Academy of Spinal Cord Injury Professionals annual meeting in New Orleans, Louisiana, 8 September 2015

Received 27 July 2015; revised 15 June 2016; accepted 21 June 2016; published online 2 August 2016 
The goal of the present study was to describe the clinical features of patients admitted to our inpatient rehabilitation unit with IRM and compare the functional outcomes of this group with those due to other causes of NTM and traumatic spinal cord injury (TSCI).

\section{MATERIALS AND METHODS}

A retrospective medical record review was performed of patients with the diagnosis of myelopathy who were discharged from a US academic inpatient rehabilitation unit between 1 January 1995 and 31 December 2010. The study population was divided into three groups on the basis of myelopathy cause: IRM, other NTM and TSCI. Because NTM and TSCI are distinct and seen in routine clinical rehabilitation practice, IRM is compared with each group to provide clinicians with a clinically relevant benchmark regarding similarities and differences of IRM.

The following data were collected on patients in all three groups: age at rehabilitation admission, gender, cause of myelopathy, completeness of injury as defined by the American Spinal Injury Association Impairment Scale, ${ }^{24}$ rehabilitation admission and discharge Functional Independence Measure (FIM) scores, ${ }^{25}$ rehabilitation length of stay (LOS) and dismissal location. Primary outcome measures used to compare all groups included total FIM change during rehabilitation and FIM change per day. Additional information collected of patients in the IRM group included presenting symptoms, infection risk factors, infection type (for example, myelitis, abscess, osteomyelitis), infectious organism, level of spinal cord injury (that is, cervical, thoracic, lumbar and cauda equina), neurologic level of impairment (paraplegia or tetraplegia), treatment (that is, antibiotics and surgical operation) and interruptions to rehabilitation stay. Several patients had pending cultures before transfer from the acute hospital setting; however, in most cases, the infectious organism was isolated before admission to inpatient rehabilitation. All patients in the IRM group were treated by an infectious disease specialist.

Means and s.d. values were calculated. Analysis of variance and pairwise comparisons were used to compare the primary outcome measures among groups with statistically significant values set at $P<0.05$ and $P<0.02$, respectively. Analysis of variance is a valid means of group comparison, despite the variation in sample size among IRM, NTM and TSCI groups. We can assume 'normality' of our sample mean using the central limit theorem that states that means will be normally distributed when each sample size is sufficient (that is, $\geqslant 30$ ). ${ }^{26}$

Table 1 Infectious pathogens of the infection-related myelopathy group

\begin{tabular}{lc} 
Infectious organism & Patients, no. (\%) \\
\hline Bacterial infection ( $\mathrm{n}=32$ ) & \\
$\quad$ Staphylococcus aureus & $19(47.5)$ \\
Escherichia coli & $2(5.0)$ \\
Lactobacillus & $1(2.5)$ \\
Streptococcus viridans & $1(2.5)$ \\
Salmonella & $1(2.5)$ \\
Enterococcus & $1(2.5)$ \\
Streptococcus pneumoniae & $1(2.5)$ \\
S. aureus+Staphylococcus epidermidis & $2(5.0)$ \\
E. coli+Streptococcus viridans+Propionibacterium acnes & $1(2.5)$ \\
Unknown, presumed bacterial & $3(7.5)$ \\
Tuberculous infection ( $\mathrm{n}=3$ ) & \\
$\quad$ Mycobacterium tuberculosis & $3(7.5)$ \\
Viral infection ( $=3$ ) & \\
$\quad$ HIV & $1(2.5)$ \\
$\quad$ West Nile virus & $2(5.0)$ \\
Viral+spirochetal infection ( $\mathrm{n}=1$ ) & \\
$\quad$ Varicella zoster+HIV+Treponema pallidum & $1(2.5)$ \\
Parasitic infection ( $=1$ ) & \\
$\quad$ Schistosoma species & $1(2.5)$ \\
\hline
\end{tabular}

Approval for this study was obtained from our institution's review board, and the approved protocol was followed. We certify that all applicable institutional and governmental regulations concerning the ethical treatment of human subjects were followed during the course of this research.

\section{RESULTS}

Over the 16-year study period, 1601 patients were discharged from our academic inpatient rehabilitation unit with a primary diagnosis of myelopathy. Of these patients, $40(2.5 \%)$ were found to have infectious causes of myelopathy and comprised the IRM group. In addition, 1105 patients $(69.0 \%)$ had NTM from another cause, and $456(28.5 \%)$ had traumatic causes of myelopathy. Because of the referral bias to our institution and the slower onset of NTM, patients with NTM are able to travel to our center for diagnosis and treatment. This accounts for the large number of NTM patients compared with TSCI patients in our study population. Of the IRM, 1.2\% $(n=3)$ occurred between 1995 and 1999, 2.9\% $(n=11)$ between 2000 and 2004 and 5.0\% $(n=26)$ between 2005 and 2010. As our study was not population based, we do not know whether the increasing percentage of cases from IRM was because of referral bias or a change in disease frequency in the population.

\section{IRM group}

Infectious pathogens were grouped according to the International Spinal Cord Society Data Sets for nontraumatic spinal cord injury, ${ }^{27}$ with the exception of classifying tuberculous infections as a separate group from bacterial. Pathogen types were bacterial $(n=32$, $80.0 \%)$, tuberculous $(n=3,7.5 \%)$, viral $(n=3,7.5 \%)$, parasitic $(n=1$, $2.5 \%)$ and more than 1 pathogen type $(n=1,2.5 \%)$ (Table 1$)$. The final patient had concomitant spirochetal and viral infections. No fungal infections were found. Infectious pathogens were identified with use of tissue or blood culture or both. Of the patients with bacterial causes, 26 were infected with a single organism, 3 with multiple organisms and 3 with negative cultures, but organisms were presumed to be of bacterial origin on the basis of imaging and surgical findings.

S. aureus was the most common organism in the bacterial group (65.6\%). Of the bacterial infections, epidural abscess was the most common infection type, affecting $91 \%$ of these patients; $53 \%$ had vertebral osteomyelitis; $50 \%$ had discitis; $38 \%$ had infections involving all sites; and 19\% had infection involving 2 sites. One patient had bacterial meningoencephalitis.

Viral causes included human immunodeficiency virus myelitis, West Nile virus myelitis and encephalomyelitis. One patient had concomitant varicella zoster myelitis, neurosyphilis and human immunodeficiency virus infections. The patients with tuberculosis had myelopathy associated with discitis $(n=1)$ or spondylitis $(n=2)$, also known as Pott's disease. The parasitic cause of myelopathy was schistosomiasis myelitis.

Twenty-five patients (62.5\%) had paraplegia and 15 (37.5\%) had tetraplegia. Among infections, $75 \%$ involved single spinal segments and $25 \%$ involved multiple spinal segments. The thoracic spine was the most commonly involved spinal segment $(n=21,52.5 \%)$. Sixteen patients had involvement of the cervical spine; 16 had involvement of the lumbar spine and 2 had involvement of the cauda equina.

Risk factors for infection were identified on the basis of those reported in prior studies ${ }^{6,11,13-16}$ (Table 2). The patient with schistosomiasis myelitis had no identifiable risk factors, but all other patients had at least one risk factor. The most common presenting symptoms included pain (60\%) and weakness (53\%). 
Table 2 Infection risk factors and presenting symptoms of the infection-related myelopathy group

\begin{tabular}{|c|c|}
\hline Variable & Patients, no. (\%) \\
\hline \multicolumn{2}{|l|}{ Infection risk factors } \\
\hline Age $>65$ years & $15(37.5)$ \\
\hline Prior nonspine infection (in past 1 month) & $12(30.0)$ \\
\hline Spine surgery ( $>1$ years ago) & $9(22.5)$ \\
\hline Nonspine malignancy & $9(22.5)$ \\
\hline Diabetes mellitus & $8(20.0)$ \\
\hline Current nicotine use & $8(20.0)$ \\
\hline Spine surgery ( $\leqslant 1$ year ago) & $6(15.0)$ \\
\hline Prior spine infection & $5(12.5)$ \\
\hline Nonspine surgery ( $\leqslant 1$ year ago) & $5(12.5)$ \\
\hline Immune suppression or corticosteroid use & $5(12.5)$ \\
\hline Decubitus or other skin ulcer & $5(12.5)$ \\
\hline Intravenous or other illicit drug use & $3(7.5)$ \\
\hline Chronic osteomyelitis & $2(5.0)$ \\
\hline Infected foreign body & $1(2.5)$ \\
\hline Spine fracture & $1(2.5)$ \\
\hline Hepatitis C & $1(2.5)$ \\
\hline Current alcohol abuse & $1(2.5)$ \\
\hline Spine malignancy & $1(2.5)$ \\
\hline \multicolumn{2}{|l|}{ Presenting symptoms } \\
\hline Pain & $24(60.0)$ \\
\hline Weakness & $21(52.5)$ \\
\hline Sensory change & $9(22.5)$ \\
\hline Fever & $7(17.5)$ \\
\hline Change in bladder function & $7(17.5)$ \\
\hline Mental status change & $3(7.5)$ \\
\hline Bacteremia & $3(7.5)$ \\
\hline Change in bowel function & $3(7.5)$ \\
\hline Movement disorder & $2(5.0)$ \\
\hline Sepsis & $1(2.5)$ \\
\hline Imaging finding & $1(2.5)$ \\
\hline Skin erythema & $1(2.5)$ \\
\hline
\end{tabular}

All patients in the IRM group had evidence of acute infection, with one exception. This sole patient had acute myelopathy secondary to progressive structural spine destruction because of Pott's disease.

All patients with bacterial infections received initial intravenous antibiotic treatment, and 59\% received oral antibiotics following completion of intravenous therapy. Disease-specific treatment was performed for the patients with syphilis, schistosomiasis and active tuberculosis. Twenty-eight patients with bacterial infections (88\%) and 2 of the 3 patients with tuberculosis underwent surgical intervention.

The rehabilitation courses of seven patients were interrupted because of acute medical issues. This subset contained six patients with bacterial infections and one patient with active tuberculosis. Reasons for these interruptions included psychiatric hospitalization for suicidal ideation $(n=1)$, wound dehiscence $(n=1)$, worsening neurologic deficits requiring unplanned surgical intervention $(n=2)$, planned surgical interventions with 1 related and 1 unrelated to myelopathy $(n=2)$ and mental status change because of narcotic medication $(n=1)$. The patient with wound dehiscence did not return to the rehabilitation unit following surgical intervention. The remainder of the patients returned to the unit, and their lengths of rehabilitation interruption ranged from 4 to 30 days.

\section{All groups}

The IRM group included myelopathy due to infections of any type. The NTM group included other NTMs, as defined by the International Spinal Cord Society non traumatic SCI Data Set. ${ }^{27}$ The most common causes in the NTM group were degenerative disease (41\%), tumor (32\%), vascular disease (12\%), inflammatory disease $(5 \%)$ and congenital disease $(4 \%)$. The TSCI group contained patients with any cause of traumatic myelopathy.

Table 3 summarizes the demographic data for all groups, including age, gender and completeness of injury. The IRM and NTM groups were significantly older than the TSCI group $(P<0.001)$. The majority of patients in each group were male and had incomplete injuries, measured at rehabilitation discharge.

The IRM group had significantly longer inpatient rehabilitation LOS (mean 31, 95\% confidence interval 25-37 days; $P<0.001$ ) than the NTM group (mean 16 days), but had no difference from the TSCI group (mean 31 days). The IRM group had significantly lower admission and discharge FIM scores and lower FIM change per day than the NTM group (Table 4). Functional status, measured by FIM scores, was not significantly different between the IRM and TSCI groups. Among all groups, no significant differences were found in total FIM change during rehabilitation. The majority of patients in each group were discharged home following inpatient rehabilitation (IRM, 62.5\%; NTM, 59.0\%; and TSCI, 74.1\%).

\section{DISCUSSION}

Our study validates many clinical characteristics and patterns of recovery previously reported for patients with IRM in developed countries and adds to the growing number of studies comparing NTM and TSCI. It also provides a unique contribution to the literature by comparing demographic characteristics and outcomes of IRM vs other causes of NTMs.

Compared with the other causes of NTM, patients with IRM had more severe functional impairments on rehabilitation admission and discharge and longer inpatient rehabilitation LOS. Despite a greater initial degree of impairment, patients with IRM had a similar degree of functional improvement as patients with other causes of NTM, and the majority of patients in both groups were discharged home following inpatient rehabilitation. Only one other study by New and Astrakhantseva $^{6}$ has compared infectious myelopathy with other causes of NTM and has found similar improvements in functional outcomes.

When comparing functional outcomes between our IRM and TSCI groups, we found admission and discharge FIM scores, LOS and discharge destination to be similar between the two groups. As a result, it may be reasonable for clinicians to use TSCI outcomes at their institution to estimate outcomes for patients with IRM. Multiple prior studies have compared IRM and TSCI, with varied results. ${ }^{14-16}$ Zafonte et al. ${ }^{14}$ compared myelopathy due to epidural abscess with TSCI and found significant functional improvements in both groups; however, the TSCI group had a greater degree of improvement. The traumatic group in that study also had significantly longer inpatient rehabilitation LOS (mean 39.5 days) than the epidural abscess group (mean 27.3 days; $P<0.01$ ). The majority of patients in both groups were discharged home.

Koo et al. ${ }^{15}$ in British Columbia also compared myelopathy due to epidural abscess with TSCI. They found the epidural abscess group had lower admission functional motor scores but showed a significant trend $(P=0.047)$ toward greater neurologic improvement in this group. In contrast, McKinley et al. ${ }^{16}$ compared IRM of any type with TSCI in the United States. Their study found no significant difference 
Table 3 Demographic characteristics and neurologic level of injury of all groups

\begin{tabular}{|c|c|c|c|}
\hline \multirow[t]{2}{*}{ Characteristic } & \multicolumn{3}{|c|}{ Group $(\mathrm{N}=1601)$} \\
\hline & $\operatorname{IRM}(\mathrm{n}=40)$ & NTM $(\mathrm{n}=1105)$ & $T S C I(n=456)$ \\
\hline Age, mean (s.d.), years ${ }^{a}$ & $58.6(15.7)$ & $62.9(16.2)$ & $45.6(19.9)$ \\
\hline \multicolumn{4}{|l|}{ Sex, \% } \\
\hline Male & 72.5 & 59.8 & 72.4 \\
\hline Female & 27.5 & 40.2 & 27.6 \\
\hline \multicolumn{4}{|c|}{ Neurologic level of injury, $\%^{\mathrm{b}}$} \\
\hline Complete & 12.5 & 4.8 & 30.3 \\
\hline Incomplete & 87.5 & 95.2 & 69.7 \\
\hline
\end{tabular}

Abbreviations: IRM, infection-related myelopathy; NTM, nontraumatic myelopathy;

$\mathrm{TSCl}$, traumatic spinal cord injury.

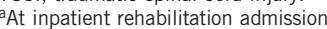

${ }^{\mathrm{A}}$ At inpatient rehabilitation discharge.

Table 4 Comparison of outcome measures of IRM and NTM

\begin{tabular}{lccr}
\hline Characteristic & IRMa $(\mathrm{n}=40)$ & NTM $(\mathrm{n}=1105)$ & P-value \\
\hline LOS, mean (95\% CI) & $31(25-37)$ & $16(15-17)$ & $<0.001$ \\
Admission FIM, mean (95\% CI) & $51(44-57)$ & $63(62-64)$ & 0.001 \\
Discharge FIM, mean $(95 \% \mathrm{CI})$ & $75(67-83)$ & $87(86-89)$ & 0.005 \\
FIM change/day, mean $(95 \% \mathrm{Cl})$ & $1.3(0.7-1.9)$ & $2.2(2.1-2.4)$ & 0.002 \\
\hline
\end{tabular}

Abbreviations: $\mathrm{Cl}$, confidence interval; FIM, Functional Independence Measure; IRM, infectionrelated myelopathy; LOS, length of stay (days) in inpatient rehabilitation; NTM, nontraumatic myelopathy.

aThere was no significant difference in FIM scores between IRM and TSCI.

among rehabilitation LOS, admission or discharge FIM scores between the two groups; however, the IRM group had significantly lower FIM score changes $(P<0.05)$, lower FIM score change per day $(P<0.05)$ and was less likely to be discharged home following inpatient rehabilitation. Despite these findings, $>50 \%$ of patients in the IRM group were discharged home vs $>70 \%$ in the TSCI group.

Other studies of IRM outcomes performed without comparison groups have also found positive outcomes. Yen et al. ${ }^{11}$ studied pyogenic and tuberculous causes of myelopathy in Singapore and found both groups had significant functional gains with activities of daily living and mobility during rehabilitation $(P<0.05)$, with the majority discharged home. LOS on the inpatient rehabilitation unit in their study ranged from 15 to 150 days (mean 73 days). New et al. ${ }^{2}$ and New and Astrakhantseva ${ }^{6}$ in Australia studied spinal IRM of all causes and found these patients made significant FIM motor score improvements $(P<0.001)$ during rehabilitation, with lengths of stay ranging from 65 to 135 days (mean 106 days). The authors noted that unlike the US health-care system, there is no limit for LOS on their inpatient rehabilitation unit and their patients tend to have longer LOS than those in the United States. This factor may contribute to their findings of higher FIM motor scores at discharge and a greater percentage of patients discharged home. ${ }^{6}$

Demographic characteristics of our IRM group, including age and gender, mostly confirm those of prior studies ${ }^{6,11,13-16}$ with a couple of exceptions. Morillo-Leco et al. ${ }^{12}$ in Spain and Nas et al. ${ }^{28}$ in Turkey studied populations with a higher proportion of cases due to spinal tuberculosis and found their subjects to be younger, with mean age at admission of 37.3 and 37.9 years, respectively. Infection risk factors in our study were consistent with prior literature, with the exception that our population had a lower proportion of intravenous and illicit drug use, ${ }^{6,11,14-16}$ likely reflecting the sociodemographic characteristics of our regional population.

We add to the growing body of evidence that patients with IRM present most commonly with pain and weakness from an epidural abscess and this results in incomplete paraplegia. ${ }^{6,11,16,21}$ Our study validates previous studies performed by Yen et al. ${ }^{11}$ Nas et al. ${ }^{28}$ Weingarden and Swarczinski, ${ }^{13}$ Curry et al. ${ }^{23}$ and McKinley et al. ${ }^{16}$ that found the thoracic region to be most commonly involved. This outcome seems to be an unresolved issue, however, because Koo et al. ${ }^{15}$ found the majority of patients with IRM had cervical level, complete injuries, and Ribeira et al. ${ }^{17}$ Connor et al. ${ }^{7}$ and Patel et al. ${ }^{22}$ found the most common level of involvement to be the lumbar spine. Few studies have evaluated the effect of level on outcome, however, Zafonte et al. ${ }^{14}$ found that patients with thoracic and lumbar lesions had greater FIM motor gains, but the finding was not statistically significant $(P=0.13)$. In our population, as well as many prior study populations, the most common infectious organism isolated was $S$. aureus. $6,7,11-13,15,16,22$ Pathogens differ geographically, however, as tuberculosis continues to be a prevalent cause of IRM in Africa, India and Singapore. ${ }^{5,11,17-20}$ Shweikeh et al. ${ }^{21}$ recently conducted a review of incidence and management of bacterial epidural abscesses and emphasized the importance of early diagnosis and treatment of this condition, with reported mortality rates between 5 and $23 \%$ and morbidity rates between 33 and $47 \%$. In our series, $75 \%(n=30)$ underwent surgical intervention. Worldwide, the proportion of patients treated operatively varies considerably, ranging from $37 \%$ to $92 \% .{ }^{6,11,13,15,21,22,28}$ Studies have suggested that such a wide range in management may be attributed to variation in health-care systems, access to health care, allocation bias, available resources, patient comorbidities and extent of the lesion., ${ }^{4,21}$ Management also seems to vary because of infectious etiologic factors, with a higher proportion of patients with tuberculosis of the spine undergoing surgical intervention. ${ }^{11,17}$ The majority of recent studies suggest antibiotic therapy and surgical management within $72 \mathrm{~h}$ of the onset of neurologic deficits in patients with comorbidities that place them at high risk of having medical management fail, such as diabetes, age $>65$ years and cultures positive for methicillin-resistant S. aureus. ${ }^{21,23}$

Our study is, to our knowledge, the first investigation to compare functional outcomes of IRM with both other nontraumatic and traumatic myelopathy populations. Although there were significant age differences, the IRM group had similar levels of functional impairment, achieved the same degree of functional improvement during inpatient rehabilitation and had similar lengths of stay as our TSCI population. Despite a higher degree of functional limitation compared with other causes of NTM, our patients with infectious causes of myelopathy achieved the same degree of functional improvement as other causes of NTM, although over a longer time spent in rehabilitation.

Our study has a number of limitations worth noting. Its design is a retrospective cohort, causing some limitations in the availability of data. It has a small sample size; however, it is one of the larger studies regarding rehabilitation outcomes for IRM. Similar to other tertiary centers, our center is subject to referral bias. Despite these limitations, our study validates the findings of prior studies and extends them through our comparison to all other NTMs.

Additional studies are necessary to determine the long-term prognosis in IRM, and prospective studies are needed to identify factors predictive of outcomes, such as ambulation. Although challenging, a study investigating the role of surgical intervention would also be of interest. 


\section{CONCLUSION}

Infectious etiologies have been found to comprise between 3 and $49 \%$ of the NTMs ${ }^{1-5}$ and represent a unique entity with distinct recovery patterns and outcomes. In our cohort, patients with infectious myelopathy were similar to patients with TSCI with regard to functional impairment and outcome. Patients with IRM achieved a similar degree of functional improvement during inpatient rehabilitation as the NTM cohort, although over a longer period. Future investigations should study this population prospectively with long-term follow-up to improve our collective knowledge to better educate patients, families and medical providers regarding the long-term outcomes of IRM.

\section{DATA ARCHIVING}

There were no data to deposit.

\section{CONFLICT OF INTEREST}

The authors declare no conflict of interest.

1 McKinley WO, Seel RT, Hardman JT. Nontraumatic spinal cord injury: incidence, epidemiology, and functional outcome. Arch Phys Med Rehabil 1999; 80: 619-623.

2 New PW, Rawicki HB, Bailey MJ. Nontraumatic spinal cord injury: demographic characteristics and complications. Arch Phys Med Rehabil 2002; 83: 996-1001.

3 Vervoordeldonk JJ, Post MW, New P, Clin Epi M, Van Asbeck FW. Rehabilitation of patients with nontraumatic spinal cord injury in the Netherlands: etiology, length of stay, and functional outcome. Top Spinal Cord Inj Rehabil 2013; 19: 195-201.

4 New PW, Reeves RK, Smith E, Townson A, Eriks-Hoogland I, Gupta A et al. International retrospective comparison of inpatient rehabilitation for patients with spinal cord dysfunction epidemiology and clinical outcomes. Arch Phys Med Rehabil 2015; 96: 1080-1087.

5 Catz A, Goldin D, Fishel B, Ronen J, Bluvshtein V, Gelernter I. Recovery of neurologic function following nontraumatic spinal cord lesions in Israel. Spine (Phila Pa 1976) 2004; 29: 2278-2282.

6 New PW, Astrakhantseva I. Rehabilitation outcomes following infections causing spinal cord myelopathy. Spinal Cord 2014; 52: 444-448.

7 Connor DE Jr, Chittiboina P, Caldito G, Nanda A. Comparison of operative and nonoperative management of spinal epidural abscess: a retrospective review of clinical and laboratory predictors of neurological outcome. J Neurosurg Spine 2013; 19: $119-127$.
8 Carey M. Infections of the spine and spinal cord. In: Youmans JR (ed). Neurological Surgery 4th edn. Saunders: Philadelphia, PA. 1996, pp 3275-3304.

9 Allen M, Flannery A, Fischer J. Spinal epidural and subdural abscesses. In: Wilkins RH, Rengachary SS (eds). Neurosurgery 2nd edn. McGraw-Hill: New York, NY, USA, 1996, pp 3327-3329.

10 Darouiche RO. Spinal epidural abscess. N Engl J Med 2006; 355: 2012-2020.

11 Yen HL, Kong KH, Chan W. Infectious disease of the spine: outcome of rehabilitation. Spinal Cord 1998; 36: 507-513.

12 Morillo-Leco G, Alcaraz-Rousselet MA, Diaz-Borrego P, Saenz-Ramirez L, Artime C, Labarta-Bertol C. Clinical characteristics of spinal cord injury caused by infection. Rev Neurol 2005; 41: 205-208.

13 Weingarden SI, Swarczinski C. Non-granulomatous spinal epidural abscess: a rehabilitation perspective. Paraplegia 1991; 29: 628-631.

14 Zafonte RD, Ricker JH, Hanks RA, Wood DL, Amin A, Lombard L. Spinal epidural abscess: study of early outcome. J Spinal Cord Med 2003; 26: 345-351.

15 Koo DW, Townson AF, Dvorak MF, Fisher CG. Spinal epidural abscess: a 5-year case-controlled review of neurologic outcomes after rehabilitation. Arch Phys Med Rehabil 2009; 90: 512-516.

16 McKinley W, Merrell C, Meade M, Brooke K, DiNicola A. Rehabilitation outcomes after infection-related spinal cord disease: a retrospective analysis. Am J Phys Med Rehabil 2008; 87: 275-280.

17 Ribeira T, Veiros I, Nunes R, Martins L. Spondilodyscitis: five years of experience in a department of rehabilitation. Acta Med Port 2008; 21: 559-566.

18 Draulans N, Kiekens C, Roels E, Peers K. Etiology of spinal cord injuries in Sub-Saharan Africa. Spinal Cord 2011; 49: 1148-1154.

19 Gupta A, Taly AB, Srivastava A, Murali T. Non-traumatic spinal cord lesions: epidemiology, complications, neurological and functional outcome of rehabilitation. Spinal Cord 2009; 47: 307-311.

20 Hart C, Williams E. Epidemiology of spinal cord injuries: a reflection of changes in South African society. Paraplegia 1994; 32: 709-714.

21 Shweikeh F, Saeed K, Bukavina L, Zyck S, Drazin D, Steinmetz MP. An institutional series and contemporary review of bacterial spinal epidural abscess: current status and future directions. Neurosurg Focus 2014; 37: E9.

22 Patel AR, Alton TB, Bransford RJ, Lee MJ, Bellabarba CB, Chapman JR. Spinal epidural abscesses: risk factors, medical versus surgical management, a retrospective review of 128 cases. Spine J 2014; 14: 326-330.

23 Curry WT Jr, Hoh BL, Amin-Hanjani S, Eskandar EN. Spinal epidural abscess: clinical presentation, management, and outcome. Surg Neurol 2005; 63: 364-371.

24 Kirshblum S, Waring W 3rd. Updates for the International Standards for Neurological Classification of Spinal Cord Injury. Phys Med Rehabil Clin N Am 2014; 25: 505-517.

25 Keith RA, Granger CV, Hamilton BB, Sherwin FS. The functional independence measure: a new tool for rehabilitation. Adv Clin Rehabil 1987; 1: 6-18.

26 Daniel WW. Biostatistics: A Foundation for Analysis in the Health Sciences 9th edn. John Wiley \& Sons, Inc.: Hoboken, NJ, 2009.

27 New PW, Marshall R. International Spinal Cord Injury Data Sets for non-traumatic spinal cord injury. Spinal Cord 2014; 52: 123-132.

28 Nas K, Kemaloglu MS, Cevik R, Ceviz A, Necmioglu S, Bukte Y et al. The results of rehabilitation on motor and functional improvement of the spinal tuberculosis. Joint Bone Spine 2004; 71: 312-316. 